\title{
Valores de la constante $B$ del método de resistencia de polarización para veinte sistemas metal-medio diferentes ${ }^{(\bullet)}$
}

\author{
J.A. González ${ }^{(*)}$, J. Albéniz ${ }^{(*)}$ y S. Feliu ${ }^{(*)}$ \\ Resumen En una investigación sobre las posibilidades de aplicación de las técnicas electroquímicas a pie de \\ obra, se utilizaron los métodos de resistencia de polarización y de intersección, sobre pequeñas pro- \\ betas en el laboratorio, como procedimientos de contraste. Los ensayos se extendieron a veinte siste- \\ mas metal-medio diferentes, cubriendo una amplia gama de resistividades del medio y de resistencia \\ a la corrosión del material metálico. Se ofrece una recopilación de las pendientes de Tafel para todos \\ los sistemas, que permite obtener un pequeño catálogo de valores de la constante $B$ de la fórmula de \\ Stern y Geary $\left(i_{\text {corr }}=B / R_{\mathrm{p}}\right)$. \\ Palabras clave: Resistencia de polarización. Curvas de polarización. Constante B. Acero. \\ Cobre. Cinc. Acero inoxidable. Medios agresivos.

\section{Polarization resistance constant $B$ values for 20 different metal- environment systems}

\begin{abstract}
In a research about the possibilities of the on-site application of electrochemical techniques, the methods of polarization resistance and intersection were utilised on small metal specimens in lab studies as contrast procedure. The tests encompassing 20 different metal-environment systems, covering a wide variety of environments resistivities and corrosion resistance of metallic material. A compilation of the Tafel slopes for all the considered systems is given, which enables to obtain a small catalog of constant $B$ in the Stern-Geary formula $\left(i_{\text {corr }}=B / R_{\mathrm{p}}\right)$.
\end{abstract}

Keywords: Polarization resistance. Polarization curves. Constant B. Steel. Copper. Zinc. Stainless steel. Aggressive media.

\section{INTRODUCCIÓN}

La estimación de las velocidades instantáneas de corrosión por medida de la resistencia de polarización:

$$
i_{\text {corr }}=\frac{b_{\mathrm{a}} \cdot b_{\mathrm{c}}}{2,3\left(b_{\mathrm{a}}+b_{\mathrm{c}}\right)} \cdot \frac{\Delta I}{\Delta E}=\frac{B}{R_{\mathrm{p}}}
$$

\footnotetext{
$(\bullet$ Trabajo recibido el día 16 de mayo de 1995.

(*) Centro Nacional de Investigaciones Metalúrgicas (CSIC). Avda. Gregorio del Amo, 8. 28040-Madrid (España).

(**) Escuela Universitaria de Ingenieros Técnicos Industriales de Madrid. Ronda de Valencia, 3. 28012Madrid (España).
}

reúne tantas ventajas prácticas que se ha convertido en un método universalmente utilizado, a pesar de que se trata de un procedimiento inexacto, basado en una relación "aproximadamente lineal" (de ahí el nombre de "método de polarización lineal", con el que también es conocido) entre la polarización aplicada y la respuesta en corriente, en el entorno del potencial de corrosión, $E_{\text {corr }}$.

Entre las limitaciones principales del método de resistencia de polarización, reconocidas desde sus comienzos (1), aparte de su carácter de procedimiento aproximado, están las debidas al desconocimiento de las pendientes de Tafel, $b_{\mathrm{a}}$ y $b_{\mathrm{c}}$ y su posible variación con el tiempo, lo que condiciona el valor de la constante $B$.

El margen de variabilidad de las pendientes de Tafel no es muy amplio y, además, el hecho de 
encontrarse en el numerador y denominador restringe aún más el rango de valores posibles de $B$ que, en la mayoría de los casos, están comprendidos entre 0,013 y $0,052 \mathrm{~V}$ (2). Esto quiere decir que, adoptando un valor medio entre los posibles, sin ninguna información previa sobre el sistema, se puede determinar la velocidad de corrosión con un factor de error de 2, como máximo, suficientemente aproximado para muchas aplicaciones.

Este factor de error de 2 puede reducirse considerablemente cuando se conoce la electroquímica del sistema, lo que permitiría una estimación teórica de las pendientes de Tafel $\left(b_{\mathrm{a}}=2,3 \cdot R T / \alpha \mathrm{nF}\right.$ y $b_{\mathrm{c}}$ $=2,3 \cdot R T / \beta \mathrm{nF})(2)$, o bien determinando éstas experimentalmente.

El incontestable interés práctico del método de resistencia de polarización justifica, a su vez, el interés por el establecimiento de un catálogo de valores de la constante $B$, que abarque un número cada vez más amplio de sistemas, en beneficio de los usuarios de las técnicas electroquímicas, que deben establecer el valor de $B$ correspondiente a su propio sistema mediante ensayos previos, o espigar laboriosamente en una bibliografía dispersa (2-6), a pesar de algunas revisiones muy valiosas (3).

El objetivo de esta publicación es dar a conocer los valores de las pendientes de Tafel y de la constante $B$ del método de resistencia de polarización para veinte sistemas metal-medio diferentes, que cubren una amplia gama de resistividades del medio y de resistencia a la corrosión del material metálico.

\section{MATERIALES Y MÉTODOS}

Se ensayaron los veinte sistemas metal-medio resultantes de la combinación del hierro, cobre y cinc, todos ellos de pureza superior al $99 \%$ y del acero inoxidable tipo AISI 304, con agua destilada, agua potable de Madrid, solución del $3 \%$ de $\mathrm{NaCl}$ y soluciones $10^{-3} \mathrm{~N}$ de $\mathrm{NaOH}$ y $\mathrm{HCl}(7-9)$.

Como objetivo principal se pretendía realizar una prospección de las posibilidades de aplicación de las técnicas electroquímicas a pie de obra, utilizando, como métodos de contraste sobre pequeñas probetas en el laboratorio, las medidas de resistencia de polarización, el método de intersección y, cuando fue posible, determinaciones gravimétricas.

El método de intersección, que determina la $i_{\text {corr }}$ por extrapolación de los tramos rectos que, frecuentemente, exhiben las curvas de polarización en coordenadas semilogarítmicas, proporciona también valores de las pendientes de Tafel en el momento de realizar las medidas (Fig. 1), mientras que las pérdidas de peso conducen a un valor medio de $B$, combinando la fórmula [1] con la ley de Faraday:

$$
P=\frac{I_{\text {corr }} \cdot t \cdot P_{\mathrm{a}}}{n F}
$$

siendo $P$, la pérdida de peso en $\mathrm{g} ; P_{\mathrm{a}}$ el átomo gramo; $n$, la valencia; $I_{\text {corr }}$, la intensidad de corrosión para toda la probeta en $A ; t$, el tiempo en $s$; y $F$, el Faraday.

Todos los resultados se obtuvieron a temperatura ambiente, aproximadamente $25^{\circ} \mathrm{C}$, con las soluciones en reposo y durante un período de exposición de 7 días. Los datos gravimétricos corresponden a probetas de $4 \times 4 \mathrm{~cm}$, con una cara enmascarada con cinta adhesiva. Los resultados electroquímicos se obtuvieron con la técnica de 3 electrodos, delimitando sobre las probetas de $4 \times 4$ $\mathrm{cm}$, también con cinta adhesiva, un círculo de 1 $\mathrm{cm}^{2}$, que actuaba como electrodo de trabajo (ET). En las medidas de resistencia de polarización se emplearon escalones de potencial de $10 \mathrm{mV}$ y la velocidad de barrido del potencial en las curvas de polarización fue de $1 \mathrm{mV} / \mathrm{s}$, salvo que se haga constar lo contrario.

Los ensayos sólo se han realizado por duplicado, quizás porque la investigación de laboratorio se utilizó, en principio, como patrón de comparación de los ensayos sobre ET de grandes dimensiones. No obstante, aunque los niveles de confianza no sean elevados, se ha obtenido una información sistemática sobre los parámetros electroquímicos de un número apreciable de sistemas que, por las razones aducidas en la introducción, se juzga de interés dar a conocer.

\section{RESULTADOS EXPERIMENTALES}

La figura 1 muestra las curvas de polarización de los cuatro materiales ensayados en agua destilada, a los 7 días de exposición. El trazado de las curs vas es poco sensible a la velocidad de polarización, . salvo en el caso del acero inoxidable, para el que se incluyen también las curvas obtenidas a 10 y 100 $\mathrm{mV} / \mathrm{s}$.

En las figuras 2, 3, 4 y 5 se reproducen las curvas de polarización obtenidas, para condiciones similares a las de la figura 1 , en agua potable de Madrid, $\mathrm{NaCl}$ al $3 \%$, y soluciones $10^{-3} \mathrm{~N}$ de $\mathrm{NaOH}$ y $\mathrm{HCl}$, respectivamente. En el caso del acero inoxidable sólo se consideran las curvas obtenidas a 1 $\mathrm{mV} / \mathrm{s}$, igual que en los otros materiales, porque la variación con la velocidad de barrido del potencial es semejante a la que puede contemplarse en la figura 1 .

En la tabla I se ha hecho un esfuerzo sintetizador para ofrecer las pendientes de Tafel y la constante de la fórmula de Stern obtenida a partir de aquellas, 
TABLA I.- Parámetros electroquímicos de todos los sistemas estudiados, deducidos a partir de las curvas de polarización obtenidas con las células electroquímicas clásicas, excepto la constante $B_{\mathrm{G}}$ de la fórmula de

Stern, que ha sido derivada a partir de la pérdida de peso determinadas al final de los 7 días de inmersión

TABLE I.-Electrochemical parameters for all studied systems, deduced from polarization curves obtained with traditional electrochemical measurements on small specimens, excepting the constant $\mathrm{B}_{\mathrm{G}}$ in the Stern formula, derived from weight losses determined after 7 days immersion

\begin{tabular}{|c|c|c|c|c|c|c|c|c|c|c|c|c|c|c|c|c|c|}
\hline \multirow{2}{*}{\multicolumn{2}{|c|}{ edio }} & \multicolumn{4}{|c|}{ Acero } & \multicolumn{4}{|c|}{ Cobre } & \multicolumn{4}{|c|}{ Cinc } & \multicolumn{4}{|c|}{ Acero inoxidable } \\
\hline & & & & & & & & & & & & & & & & & \\
\hline $\begin{array}{l}\text { Agua } \\
\text { destilada }\end{array}$ & $\begin{array}{l}1 \mathrm{~h} \\
1 \mathrm{~d} \\
7 \mathrm{~d}\end{array}$ & $\begin{array}{r}140 \\
130 \\
68\end{array}$ & $\begin{array}{c}330 \\
\infty \\
600\end{array}$ & $\begin{array}{l}43 \\
56 \\
30\end{array}$ & 64 & $\begin{array}{r}116 \\
66 \\
68\end{array}$ & $\begin{array}{l}\infty \\
\infty \\
\infty\end{array}$ & $\begin{array}{l}50 \\
29 \\
30\end{array}$ & 29 & $\begin{array}{r}68 \\
530 \\
260\end{array}$ & $\begin{array}{l}\infty \\
\infty \\
\infty\end{array}$ & $\begin{array}{r}30 \\
230 \\
114\end{array}$ & 154 & $\begin{array}{l}330 \\
300 \\
450\end{array}$ & $\begin{array}{l}250 \\
170 \\
220\end{array}$ & $\begin{array}{l}62 \\
46 \\
64\end{array}$ & - \\
\hline $\begin{array}{l}\text { Agua } \\
\text { potable } \\
\text { de Madrid }\end{array}$ & $\begin{array}{l}1 \mathrm{~h} \\
1 \mathrm{~d} \\
7 \mathrm{~h}\end{array}$ & $\begin{array}{l}260 \\
284 \\
236\end{array}$ & $\begin{array}{c}\infty \\
\infty \\
405\end{array}$ & $\begin{array}{r}113 \\
123 \\
65\end{array}$ & 41 & $\begin{array}{r}53 \\
42 \\
130\end{array}$ & $\begin{array}{l}470 \\
320 \\
205\end{array}$ & $\begin{array}{l}21 \\
15 \\
40\end{array}$ & 19 & $\begin{array}{r}95 \\
130 \\
120\end{array}$ & $\begin{array}{l}305 \\
270 \\
260\end{array}$ & $\begin{array}{l}31 \\
38 \\
36\end{array}$ & 148 & $\begin{array}{l}340 \\
350 \\
530\end{array}$ & $\begin{array}{l}160 \\
200 \\
410\end{array}$ & $\begin{array}{r}47 \\
67 \\
100\end{array}$ & - \\
\hline $\begin{array}{l}\mathrm{NaCl}, \\
3 \%\end{array}$ & $\begin{array}{l}1 \mathrm{~h} \\
1 \mathrm{~d} \\
7 \mathrm{~d}\end{array}$ & $\begin{array}{r}100 \\
63 \\
58\end{array}$ & $\begin{array}{l}\infty \\
\infty \\
\infty\end{array}$ & $\begin{array}{l}43 \\
27 \\
25\end{array}$ & 19 & $\begin{array}{l}60 \\
60 \\
74\end{array}$ & $\begin{array}{c}\infty \\
480 \\
\infty\end{array}$ & $\begin{array}{l}26 \\
20 \\
32\end{array}$ & 23 & $\begin{array}{r}32 \\
42 \\
.79\end{array}$ & $\begin{array}{l}\infty \\
\infty \\
\infty\end{array}$ & $\begin{array}{l}13 \\
18 \\
34\end{array}$ & 24 & $\begin{array}{l}400 \\
260 \\
200\end{array}$ & $\begin{array}{l}280 \\
300 \\
200\end{array}$ & $\begin{array}{l}72 \\
60 \\
43\end{array}$ & - \\
\hline $\begin{array}{l}\mathrm{NaOH}, \\
10^{-3} \mathrm{~N}\end{array}$ & $\begin{array}{l}1 \mathrm{~h} \\
1 \mathrm{~d} \\
7 \mathrm{~d}\end{array}$ & $\begin{array}{l}79 \\
77 \\
84\end{array}$ & $\begin{array}{l}\infty \\
\infty \\
\infty\end{array}$ & $\begin{array}{l}34 \\
33 \\
37\end{array}$ & 30 & $\begin{array}{l}\infty \\
\infty \\
66\end{array}$ & $\begin{array}{c}90 \\
90 \\
142\end{array}$ & $\begin{array}{l}39 \\
39 \\
20\end{array}$ & 43 & $\begin{array}{l}273 \\
126 \\
242\end{array}$ & $\begin{array}{l}\infty \\
\infty \\
\infty\end{array}$ & $\begin{array}{r}120 \\
55 \\
105\end{array}$ & 198 & $\begin{array}{l}190 \\
370 \\
505\end{array}$ & $\begin{array}{l}160 \\
166 \\
215\end{array}$ & $\begin{array}{l}38 \\
50 \\
66\end{array}$ & - \\
\hline $\begin{array}{l}\mathrm{HCl}, \\
10^{-3} \mathrm{~N}\end{array}$ & $\begin{array}{l}1 \mathrm{~h} \\
1 \mathrm{~d} \\
7 \mathrm{~d}\end{array}$ & $\begin{array}{r}170 \\
184 \\
84\end{array}$ & $\begin{array}{l}\infty \\
\infty \\
\infty\end{array}$ & $\begin{array}{l}73 \\
80 \\
37\end{array}$ & 23 & $\begin{array}{l}320 \\
170 \\
250\end{array}$ & $\begin{array}{l}320 \\
430 \\
330\end{array}$ & $\begin{array}{l}70 \\
53 \\
62\end{array}$ & 1,1 & $\begin{array}{r}74 \\
63 \\
116\end{array}$ & $\begin{array}{l}230 \\
300 \\
340\end{array}$ & $\begin{array}{l}24 \\
23 \\
38\end{array}$ & 19 & $\begin{array}{l}600 \\
200 \\
260\end{array}$ & $\begin{array}{c}\infty \\
300 \\
305\end{array}$ & $\begin{array}{l}52 \\
52 \\
61\end{array}$ & - \\
\hline
\end{tabular}

$B_{\mathrm{E}}$, o de las pérdidas de peso a los 7 días de exposición, $B_{\mathrm{G}}$. Para determinar este último valor, se transforman las pérdidas de peso en $I_{\text {corr }}$ por la ecuación [2] y deduciendo una $R_{\mathrm{p}}$ media por el método de intersección, de representaciones como las de las figuras $1-5$, se calcula $B_{\mathrm{G}}$ a partir de la ecuación [1].

\section{DISCUSIÓN}

La limitación del método de resistencia de polarización más aireada por sus detractores es su imprecisión, el factor de error de 2 está reconocido ya por Stern (1).

Un factor de error de 2 puede ser, según los casos, un certificado de nulidad o una garantía de utilidad. En el margen de penetraciones de la corrosión superior a $1 \mathrm{~mm} / \mathrm{año}$, un error del $100 \%$, en un sentido o en el opuesto, es una aproximación grosera, aunque ningún técnico cometería el desliz de tomar en consideración un material con tal comportamiento. Si se habla, en cambio, de penetraciones del orden de $1 \mu \mathrm{m} / \mathrm{año}$, o inferiores, ¿qué otro método puede competir con el de resistencia de polarización en ofrecer un factor de error máximo de 2?

Parece posible mejorar sustancialmente la confiabilidad de las medidas de $R_{\mathrm{p}}$, al menos en teoría, determinando experimentalmente la constante $B$, o bien calculándola teóricamente, cuando se conocen con precisión las reacciones que tienen lugar.

En la práctica, sin embargo, el cálculo de la constante $B$, a partir de las pendientes de Tafel, resulta condicionado por la exactitud de éstas, que depende de múltiples factores: de la naturaleza y cinética de las reacciones de electrodo, de la presencia o ausencia de oxígeno disuelto, de los inhibidores, del carácter más o menos protector de las capas de productos de corrosión, de las posibilidades de difusión de las especies iónicas disueltas, de las condiciones de reposo o movimiento del electrólito y de las variaciones de todos estos y otros factores con el tiempo (5).

En concordancia con las dificultades acabadas de mencionar, los resultados obtenidos en esta investigación no parecen garantizar, en la mayoría de los veinte sistemas considerados, un factor de 

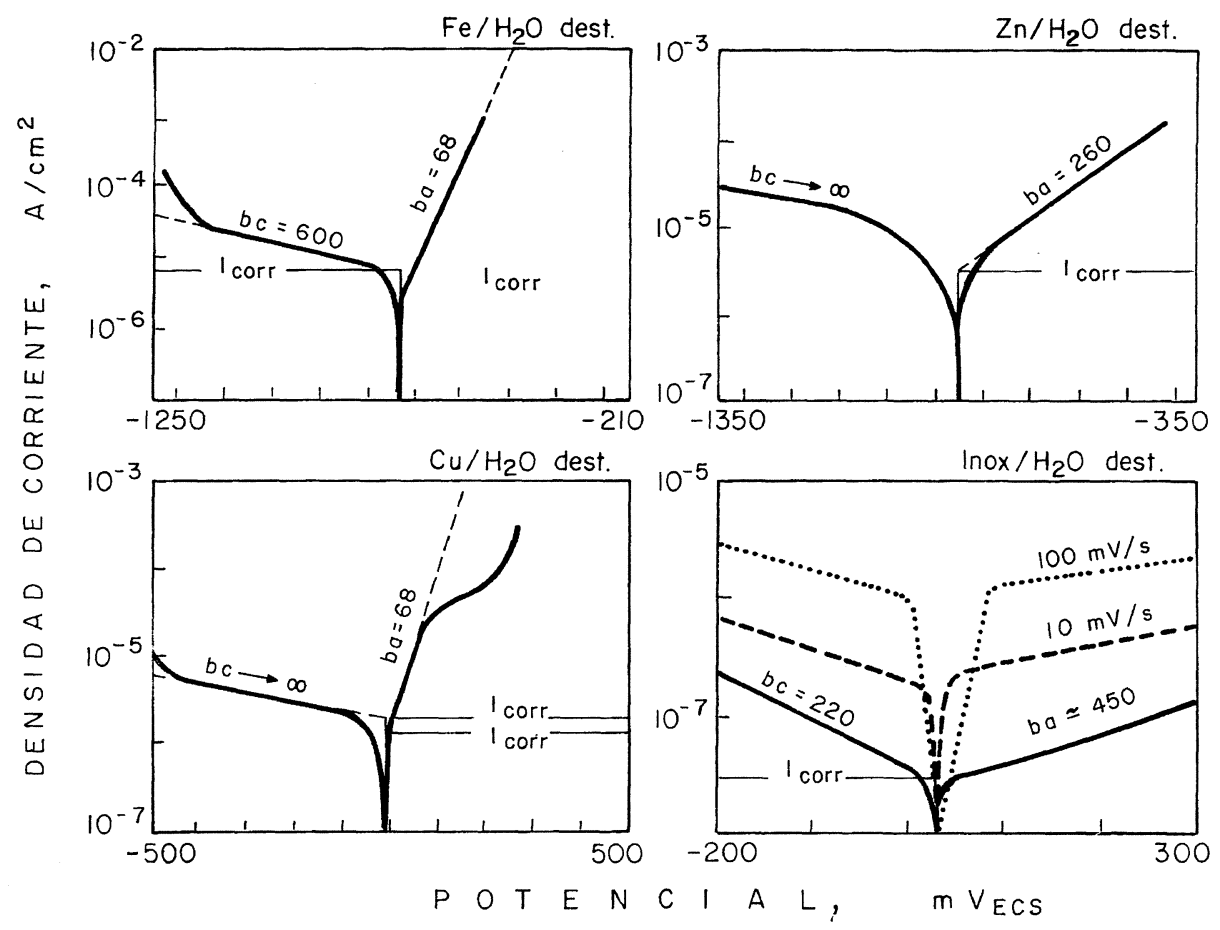

Fig. 1.- Curvas de polarización de los cuatro materiales ensayados en agua destilada, a los 7 días de exposición.

FIG. 1.- Polarization curves for the four metallic materials tested in destilled water, after 7 days immersion.
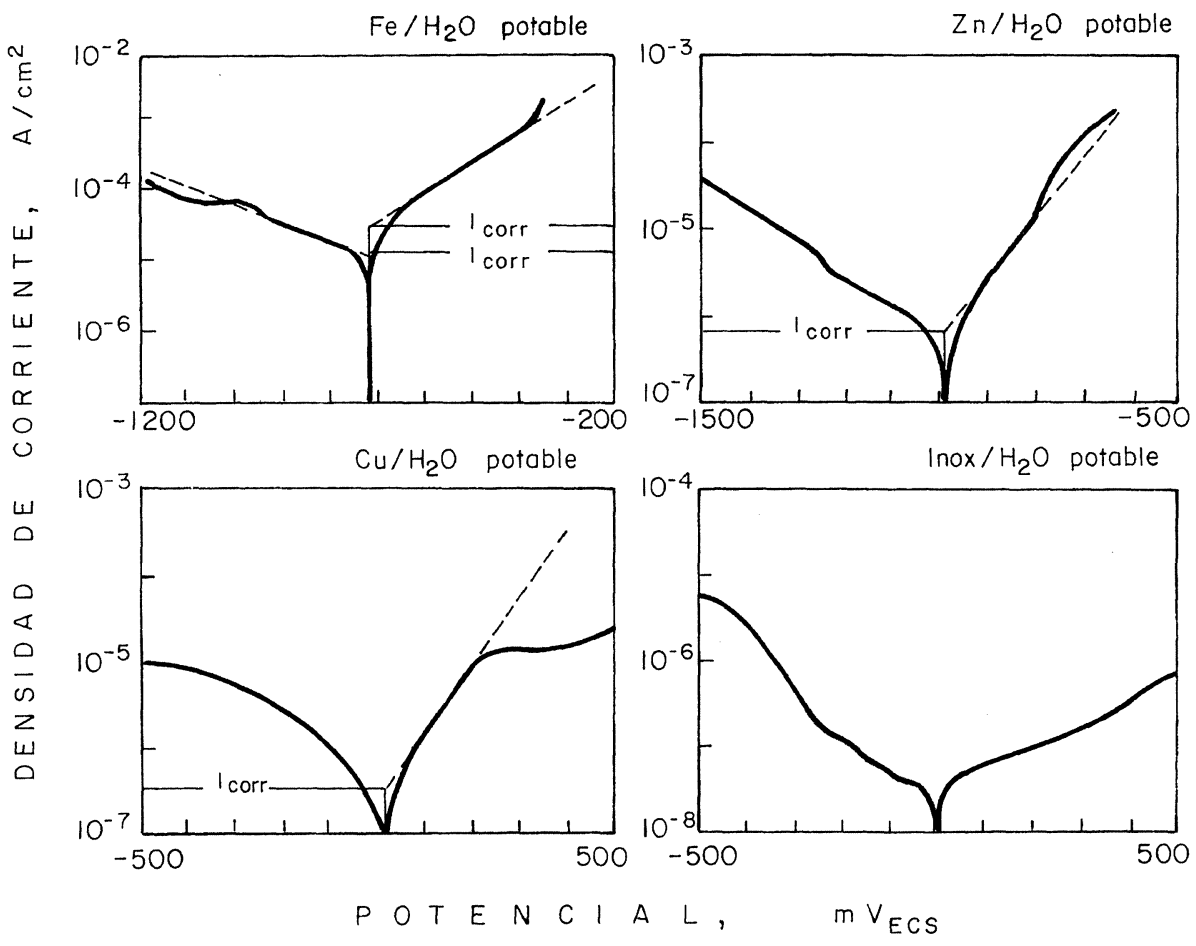

Fig. 2.- Curvas de polarización de los cuatro materiales ensayados en agua potable deMadrid, a los 7 días de exposición.

FIG. 2.- Polarization curves for the four metallic materials tested in Madrid tap water, after 7 days immersion. 


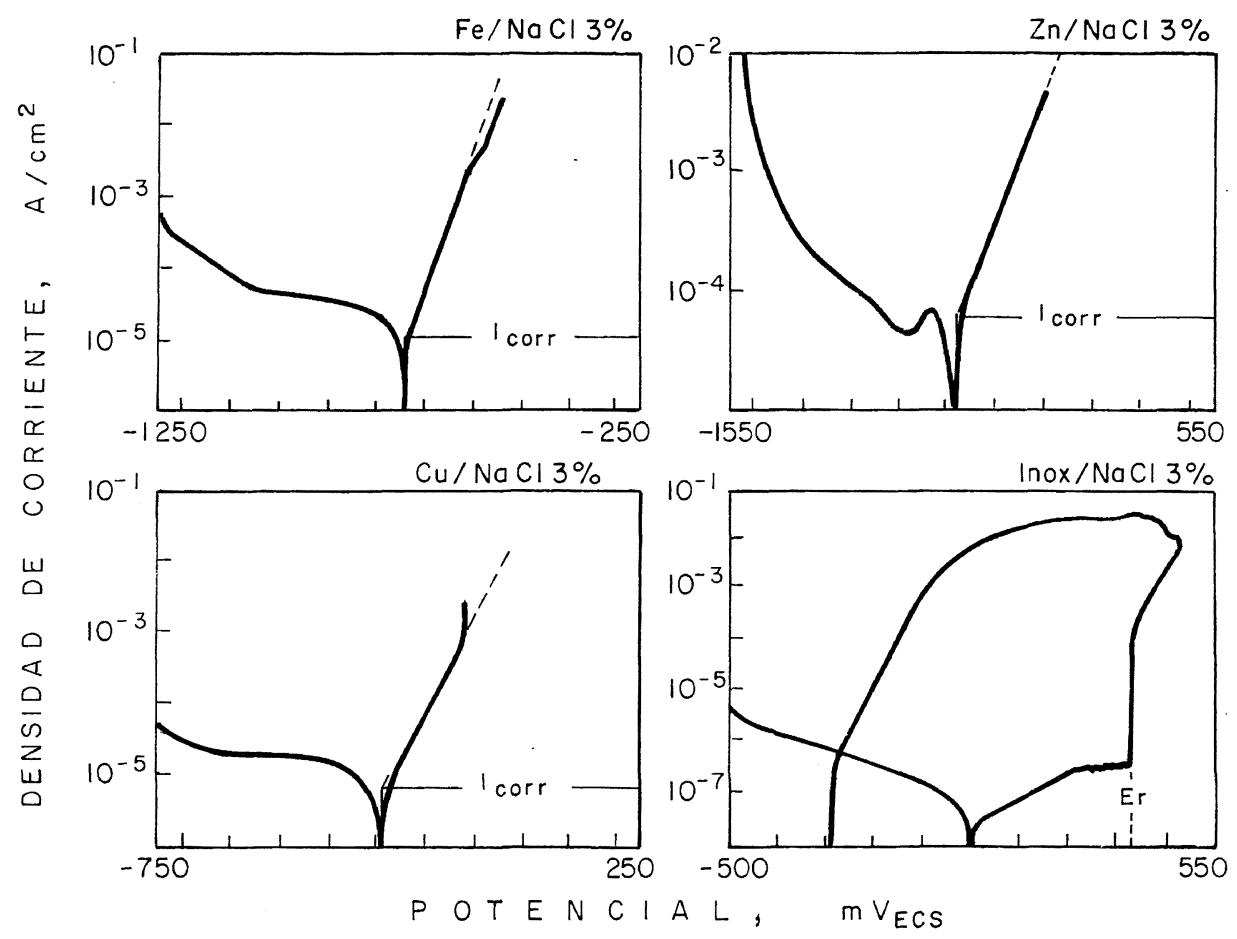

FIG. 3.- Curvas de polarización de los cuatro materiales ensayados en solución de $\mathrm{NaCl}$ al $3 \%$, a los 7 días de exposición.

FIG. 3.- Polarization curves for the four metallic materials tested in $3 \% \mathrm{NaCl}$ solution, after 7 days immersion.
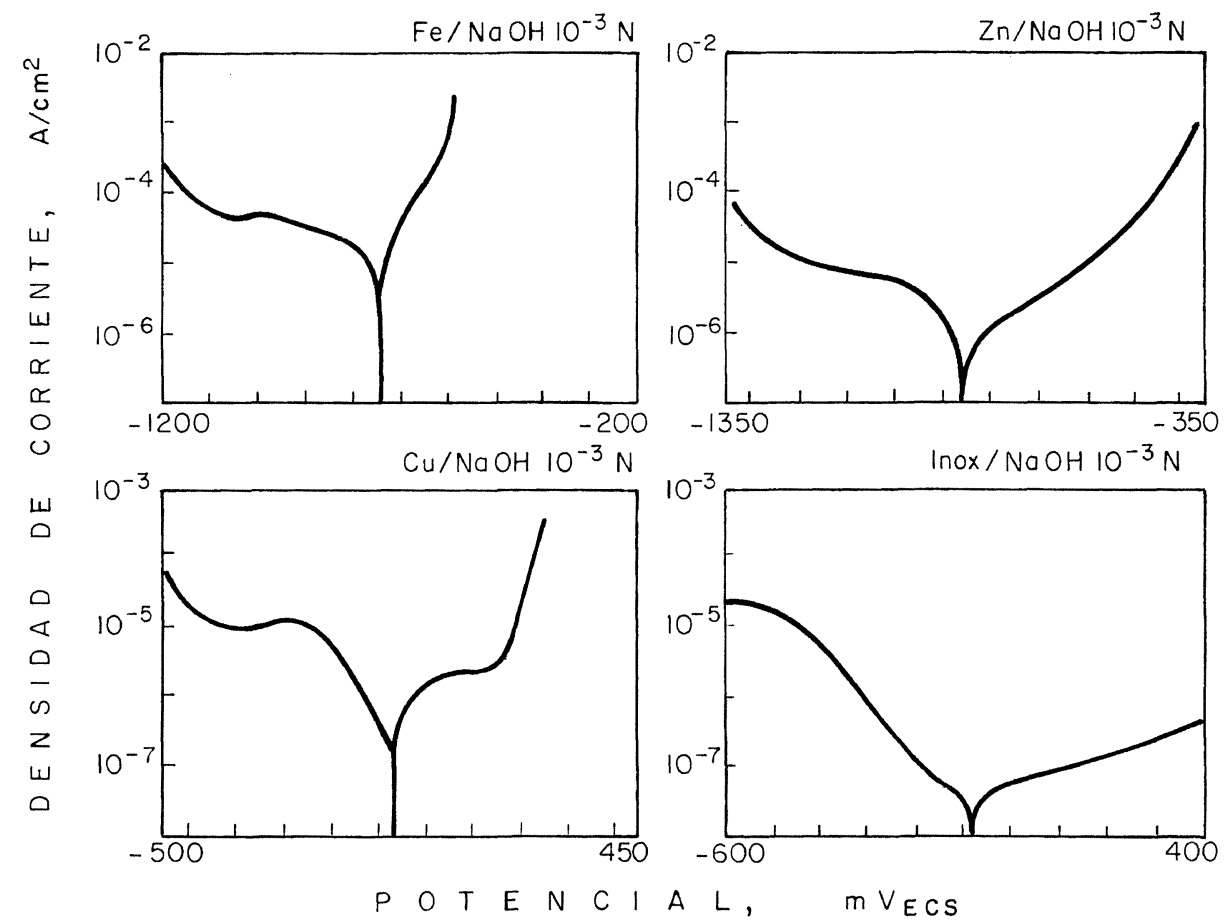

Fig. 4.- Curvas de polarización de los cuatro materiales ensayados en solución $10^{-3} \mathrm{~N}$ de hidróxido sódico, a los 7 días de exposición.

FIG. 4.- Polarization curves for the four metallic materials tested $10^{-3}$ sodium hidroxide solution, after 7 days immersion. 


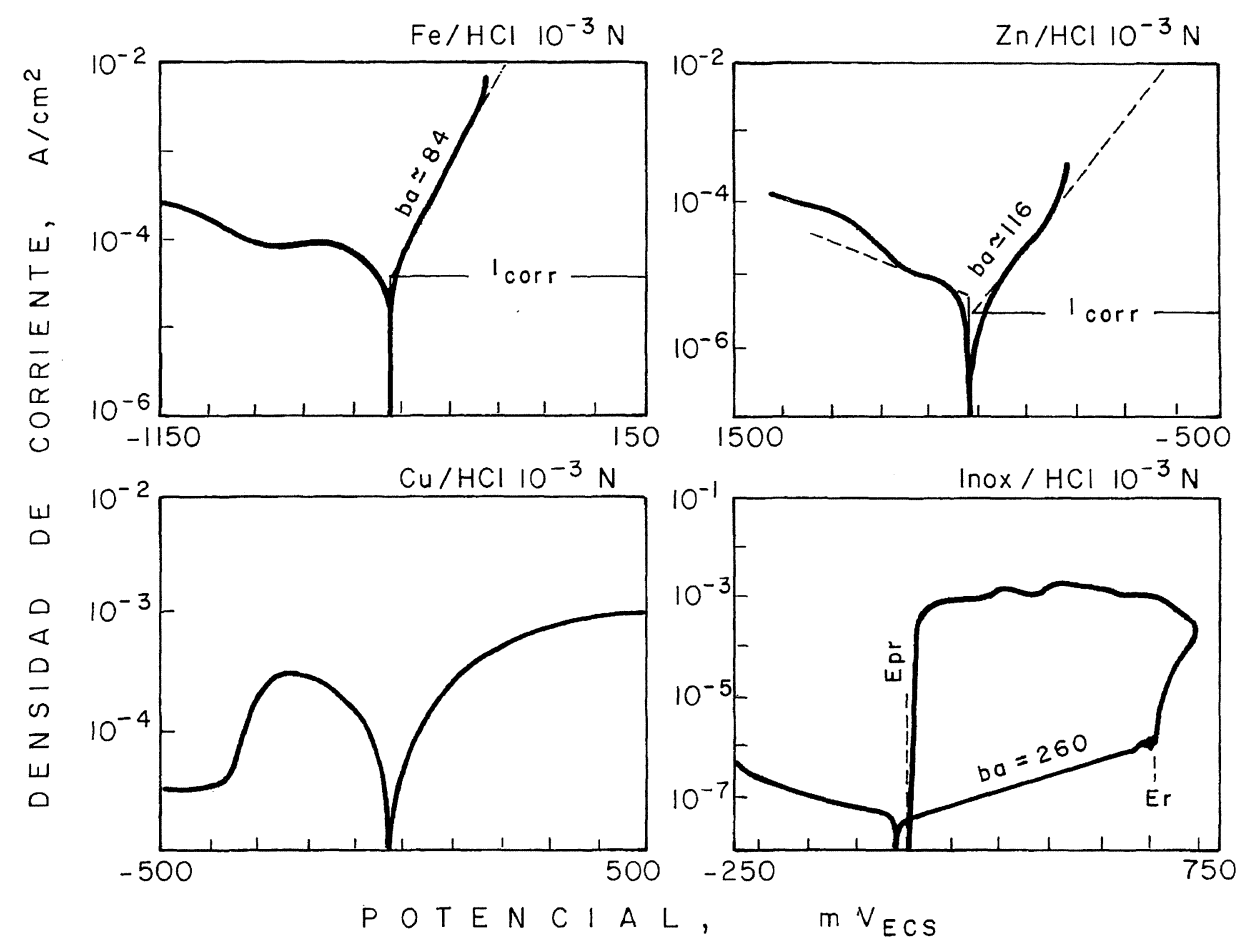

FIG. 5.- Curvas de polarización de los cuatro materiales ensayados en solución $10^{-3} \mathrm{~N}$ de ácido clorhídrico, a los 7 días de exposición.

FIG. 5.- Polarization curves for the four metallic materials tested in $10^{-3} \mathrm{~N}$ hydrochloric acid solution, after 7 days immersion.

error $\ll<2$ en la determinación de $B$, según puede deducirse de la tabla I, en la que se han incluido los valores electroquímicos de $B$ correspondientes a exposiciones de 1 h, 1 día y 7 días. Resulta evidente una considerable dispersión de los valores de $B$ con el tiempo.

Una observación detallada de las figuras 1-5, pone de relieve las peculiaridades de las curvas de polarización que restan seguridad a la determinación experimental de $B$, peculiaridades que se tratarán de resumir basándose esencialmente en la figura 1 .

a) Existe una clara tendencia hacia valores de $b_{\mathrm{c}}$ $>>b_{\mathrm{a}}$, salvo en el caso del acero inoxidable, en el que tanto $b_{\mathrm{c}}$ como $b_{\mathrm{a}}$ tienden hacia valores elevados. Pero, ¿a partir de qué valor de $b_{\mathrm{c}}$ debe admitirse una etapa controlante por difusión y adoptar un valor de $b_{\mathrm{c}}=\infty$ ?. Por ejemplo, en el sistema $\mathrm{Fe}$-agua destilada, según se proceda de una u otra forma resulta $B_{\mathrm{E}}=26 \mathrm{mV}\left(b_{\mathrm{c}}=600\right.$ $\mathrm{mV}$ y $\left.b_{\mathrm{a}}=68 \mathrm{mV}\right)$, o $B_{\mathrm{E}}=29 \mathrm{mV}\left(B_{\mathrm{E}}=b_{\mathrm{a}} / 2,3\right)$, valores casi coincidentes.

b) ¿Es más correcto suponer $b_{\mathrm{a}}=\infty$ para los materiales pasivos $\left(B_{\mathrm{E}}=b_{\mathrm{c}} / 2,3\right)$, o tomar en consideración los valores estimados gráficamente, aunque sean muy elevados?. En el sistema inoxidable-agua destilada resultan valores para $B_{\mathrm{E}}$ de 96 y $64 \mathrm{mV}$, respectivamente. Esta diferencia es aún tolerable, pero puede ser mayor en otros casos.

c) Dada la extremada dependencia del curso de las curvas de polarización, en el caso del acero inoxidable, con la velocidad de barrido del potencial, ¿qué velocidades de barrido deben utilizarse y hasta qué punto pueden conducir a resultados válidos?

d) En casos como los de los sistemas $\mathrm{Cu}$-agua de Madrid (Fig. 2), o el Cu-HCl$\cdot 10^{-3} \mathrm{~N}$ (Fig. 5), en los que no se manifiesta comportamiento de Tafel en alguna de las curvas o en ambas, ies preferible linealizar aproximadamente las curvas o adoptar un valor medio de $B$ ?

e) En el caso del cobre y del cinc, que suelen desarrollar capas protectoras, el paso de una corriente excesiva, debida por ejemplo a una sobrecompensación de la caída óhmica con el positivo feed-back del potenciostato, puede perforarlas y modificar sensiblemente las pendientes de Tafel. Las capas pasivantes del acero inoxidable suelen ser más estables, si bien se rompen también, para polarizaciones elevadas, en presencia de cloruros (Figs. 3 y 5). 
Aun reconociendo todas las imprecisiones anteriores, resaltan también una serie de hechos positivos, que ayudan a considerar en su justo valor las técnicas electroquímicas de estimación de la velocidad instantánea de corrosión, por ejemplo:

- A pesar de la evidente dispersión de los valores de $B_{\mathrm{E}}$ (Tabla I), si se comparan los datos individuales con el valor medio, sólo se supera el factor de error de 2 en el sistema Zn-agua destilada, para $1 \mathrm{~h}$ de exposición.

- Comparando los valores medios de $B_{\mathrm{G}}$ con los de $B_{\mathrm{E}}$, la relación es inferior a 2 en diez de los quince casos registrados en la tabla I, e inferior a tres en tres de los cinco casos restantes, lo que supone una más que aceptable concordancia entre los valores estimados electroquímicamente y los determinados gravimétricamente.

- Procediendo con sentido común, las distintas posibilidades señaladas en los puntos a) y b) conducen a errores muy inferiores al mencionado factor de error de 2, como se demuestra en los ejemplos allí citados.

- Con velocidades de polarización pequeñas, $<10$ $\mathrm{mV} / \mathrm{min}$, pueden realizarse estimaciones cinéticas fiables, incluso en los sistemas pasivos (punto c) (8 y 9).

- Cuando no se dan comportamientos de Tafel (punto d)), la elección de un valor intermedio de $B$, o su determinación previa por vía gravimétri$\mathrm{ca}$, parecen soluciones razonables.

- Aun en los casos conflictivos mencionados en el punto e), los resultados prueban que es posible mantenerse dentro de un margen de error aceptable.

Solamente en el sistema $\mathrm{Cu}-\mathrm{HCl} \cdot 10^{-3} \mathrm{~N}$, entre los veinte estudiados, existe una total discrepancia entre las previsiones electroquímicas y los datos de pérdida de peso, como testimonia la relación $B_{\mathrm{E}} / B_{\mathrm{G}}$ $=50-60$. La razón parece ser la presencia de un equilibrio redox en el medio, probablemente

$$
\mathrm{Cu}^{2+} \longrightarrow \mathrm{Cu}^{+}+1 \mathrm{e}^{-},
$$

contribuyendo la corriente de intercambio a la respuesta del sistema en mucha mayor medida que la corriente de corrosión. El acentuado máximo de la curva de polarización catódica (Fig. 5), evidencia la reducción de alguna sustancia del medio distinta de los protones o del oxígeno disuelto, que no muestran singularidad ninguna para el potencial del máximo. La dispersión en las medidas de $R_{\mathrm{p}}$ puede ser muy grande en este sistema, quizás dependiendo de la concentración de las especies iónicas de cobre en la solución. Las peculiaridades del comportamiento electroquímico del cobre en medios ácidos han sido destacadas ya con anterioridad (10 y 11).

Los valores de $B$ de la tabla I encajan aceptablemente, sólo aceptablemente, con los ofrecidos en (3): $21 \pm 6$ para el hierro en soluciones ácidas; $21 \pm$ 15 en soluciones de cloruros y $50 \pm 28$ para el hierro en aguas potables. Se registran más dificultades que las señaladas en el caso del cobre, quizás por las roturas locales de las capas protectoras y se comprueba la considerable dispersión que afecta a los valores de $B$ en el caso del cinc, presumiblemente a causa de las fluctuaciones de potencial, como se señala en (3), y sobre todo, a la interferencia de las capas superficiales protectoras, como también se menciona en (3), fácilmente alterables por el paso de corrientes excesivas. No obstante, parece factible, tanto en el cobre como en el cinc, a la vista de los resultados de la tabla I, una estimación electroquímica aproximada a través de medidas de $R_{\mathrm{p}}$, si se exceptúa el sistema $\mathrm{Cu}-\mathrm{HCl} \cdot 10^{-3} \mathrm{~N}$.

Por lo que se refiere al acero inoxidable, la discrepancia entre las $B$ aquí determinadas y las recogidas en la bibliografía es acusada. Se menciona un valor medio de $25 \pm 7$ (3), mientras que en la tabla I casi todos los valores están situados en el intervalo de 40-70 mV. La explicación de tan manifiestas diferencias puede radicar en que en trabajos anteriores se refieren a medios en los que el acero inoxidable permanece en el estado activo (3), mientras que en los sistemas considerados en esta investigación se mantiene siempre en el estado pasivo, hasta el punto de que resulta imposible realizar un contraste por vía gravimétrica, en un plazo de tiempo razonable.

\section{CONCLUSIONES}

- En los veinte sistemas estudiados, la determinación de la constante $B$, a partir de las pendientes de Tafel, no mejora sustancialmente la exactitud del método de resistencia de polarización.

- El factor que más contribuye a la dispersión de los valores de $B$ es la formación de las capas superficiales protectoras de productos de corrosión, susceptibles de rupturas locales por el paso de corrientes excesivas.

- A pesar de todas las dificultades, los resultados confirman la elevada confiabilidad de las medidas de $R_{\mathrm{p}}$, dentro del conocido factor de error máximo de 2 .

- En el sistema $\mathrm{Cu}-\mathrm{HCl} \cdot 10^{-3} \mathrm{~N}$, las estimaciones electroquímicas conducen a $i_{\text {corr }}$ unas 60 veces superiores a las determinadas por gravimetría, 
debido a que a la respuesta del sistema contribuye la corriente de intercambio de un equilibrio redox en mucha mayor proporción que la corriente de corrosión. No obstante, la dispersión de valores es elevada en este sistema, dependiendo de factores no bien precisados aún.

\section{REFERENCIAS}

(1) Stern, M. y Weisert, E.D. Proc. Am. Soc. Test. Mater., 59, 1959: 1280.

(2) GonZalez, J.A. "Control de la corrosión. Estudio y medida por técnicas electroquímicas". Ed. CSIC, 1989. Cap. V, pp. 101-134.

(3) Grauer, R., Moreland, P.S. y Pini, G. "A literature review of polarisation resistance constant (B) values for the measurement of corrosion rate". Repport of the European Federation of Corrosion . NACE, 1985.
(4) Mansfeld, F. Corrosion, 29, 1973: 397.

(5) Pourbaix, A. Rapports Techniques de CEBELCOR, 124, abril 1974, R.T. 219.

(6) Callow, L.M., Richardson, J.A. y Dawson, J.L. Br. Corros. J., 11, 1976: 123 y 11, 1976: 132.

(7) Albéniz, J., Gonzalez, J.A. y Feliu, S. Rev. Metal. Madrid, 26 (1), 1990: 31.

(8) Gonzalez, J.A. y Albéniz, J. Rev. Iberoam. Corros. Prot., XXI (3), 1990: 113; XXI (4), 1990: 148 y XXI (6), 1990, 211.

(9) GonZalez, J.A."Control de la corrosión. Estudio y medida por técnicas electroquímicas". Ed. CSIC, 1989. Cap. VII, pp. 173-198.

(10) PrazaK, M. Werk. Korr., 19 (10), 1968: 845.

(11) Medina, E., Pizarro, R., Salas, G., Rächle, F. y GonZALEZ, J.A. Rev. Metal. Madrid, 22 (6), 1986: 352. 